\title{
Vibration testing and evaluation for source detection of bothersome structural vibrations
}

\author{
Matthew A. Pavelchak ${ }^{1, a}$ \\ ${ }^{1}$ Walter P. Moore and Associates, Diagnostics Group, Houston, Texas, United States of America
}

\begin{abstract}
Buildings are subjected to a myriad of vibration excitation sources including mechanical systems, human activity, vehicular traffic, and other environmental conditions. The characteristics (amplitude and frequency) of the excitation from these sources can vary significantly overtime as building use patterns change, systems age, and maintenance activities vary. Overtime many building owners/operators find themselves dealing with vibration complaints which arise unexpectedly and without obvious cause. The complexity of many modern facilities can make pinpointing the source of bothersome vibrations equivalent to finding a needle in a haystack. The objective of this paper is to explore the process of vibration source detection in complex existing facilities through three recent case studies. The vibration testing and evaluation methodologies utilized for source detection on these case studies is explored to highlight challenges faced on these types of projects. Each of the case studies involve existing buildings with many years of successful operation before bothersome vibration events were reported. The case studies presented, explore complaints related to sensitive medical equipment as well as human perception of vibrations.
\end{abstract}

\section{Introduction}

Vibration concerns related to sensitive equipment and human perception often develop in existing facilities without an obvious identifiable cause after many years of acceptable performance. Pinpointing the source of these bothersome vibrations can be difficult in complex facilities such as hospitals. A variety of potential sources exist including mechanical systems, human activity, vehicular traffic and other environmental conditions. In reality the vibration excitations acting on a structure are constantly changing overtime.

Some bothersome vibrations are easy to diagnose and attribute to a nearby activity; however, the source of bothersome vibrations can sometimes be located very remote from the source of complaints. Adding to the complexity of the problem is the variability of human perception and tolerance of vibration events and the tight vibration limits under which many sensitive medical and research devices must operate.

The focus of this paper is to present a series of three case studies which explore the process used to leverage instrumentation to diagnose the sources of troublesome vibrations in complex facilities. The case studies will address the following:

1. Vibration concerns which arose in relation to highly sensitive surgical microscopes in a high-rise hospital.

2. Human perception complaints of long and intense vibration events received by building

\footnotetext{
${ }^{\mathrm{a}}$ Corresponding author: mpavelchak@walterpmoore.com
}

management in a high-rise commercial office building after 10 years of service.

3. Human perception complaints of frequent transient vibration events in a localized area of a low-rise hospital.

\section{Vibration monitoring methodology for source detection}

Complex vibration source detection testing requires the use of targeted instrumentation to quantify and characterise the nature of the ambient vibration conditions. Based upon our experience we recommend the use of an accelerometer based system with capabilities for continuous data collection and semi-real time signal display. The ability to record the full time history record of the vibration events allows for in-depth analysis of observed vibration events, while a semi-real time signal display allows for greater understanding of the nature of the vibration events in the field. The ability to record several data channels simultaneously allows for greater understanding of vibration patterns and timing within different areas of the facility of interest.

As further detailed in the case studies some testing laboratories and consultants attempt to use geophone based systems for diagnosis of human perception and sensitive equipment complaints. It is our experience that the commercially available geophone systems in the United States are not appropriate for the successful diagnosis of complex vibration problems due to lack of sensitivity, limited data recording capabilities, and 
operating modes designed to capture peak impulse results. These geophone based systems are also generally lacking in the ability to visualize the recorded vibration data in real time.

Successful source detection generally requires active monitoring of the instrumentation system by the testing team throughout the testing period to make realtime decisions regarding hypotheses. Typically potential vibration excitation sources are postulated and ranked based upon engineering judgement regarding the likelihood that each potential source is contributing to the bothersome vibrations. Then the testing team can systematically assess the impact of each potential source by observing the changes in vibration conditions at the location of interest as each source is tested. In the case of mechanical equipment, controlled cycling of each source is often possible through coordination with building maintenance personnel. Human excitation modes can be tested through coordinated activities by the testing team. In the case of external sources such as vehicular or train traffic passive observation of regular activities is often required.

Passive recording of vibration data solely for review in the office is generally ineffective in source detection projects. This data can be used to classify the duration, magnitude, and frequency spectrum of the bothersome vibrations; however, the lack of simultaneous visual observations within the building generally hampers investigation of potential sources.

\section{Case Study: Surgical microscope vibration source detection}

\subsection{Background}

A high-rise hospital in Houston, Texas, USA began to receive complaints from surgeons regarding floor mounted microscopes in the neurosurgery operating suites. The microscopes are used to guide surgeons during precise tissue removal procedures while protecting vital organs. The complaints indicated excessive distortion of the image or blurriness. Once malfunction of the microscopes was ruled out it was determined that structural vibrations were the likely cause of the microscope image quality problems. The hospital suspended use of the operating suites on the fifth floor of the facility until the issue could be resolved.

The microscopes had been in service for more than five years without incident. No published manufacturer vibration criteria were available for review; however, the offending vibrations were clearly below levels of human perception. After review of the ambient structural vibrations it was determined that the microscopes vibration criteria were best represented by the Class A criteria (2,000 Micro Inches Per Second - MIPS) presented in the American Institute of Steel Construction (AISC) Design Guide 11 (DG11).

The lower floors of the hospital were constructed with full story interstitial spaces between each level to accommodate a large quantity of mechanical equipment to support the operating rooms. Many building mechanical systems were observed in close proximity to the microscopes including air handlers, water pumps, elevators, freezers, and air compressors. Adding to the complexity, many of the surgical and laboratory spaces on the occupied floors contained dedicated mechanical systems and devices independent of the main building systems.

The building structural system is comprised of reinforced concrete columns and shear walls supporting reinforced concrete pan-joist slabs. The building had recently undergone a vertical expansion approximately doubling the building height with the addition of 12 additional stories. The construction had progressed without significant impact on the operation of the lower floors and no heavy construction was ongoing at the time of the complaints.

The building staff identified a new service elevator system in close proximity to the operating rooms which had recently been replaced as the likely cause of the vibrations.

\subsection{Testing and Results}

A tri-axial accelerometer cluster was installed on the underside of the operating room slab from the interstitial space as shown in Figure 1. This approach allowed for instrumentation without contaminating the sterile surgical suites. Initial results indicated that the principle vibrations at this location were in the vertical orientation with a peak response of approximately 7,000 MIPS at 12 Hertz (Hz). These vibrations fluctuated overtime but were generally continuous in nature.

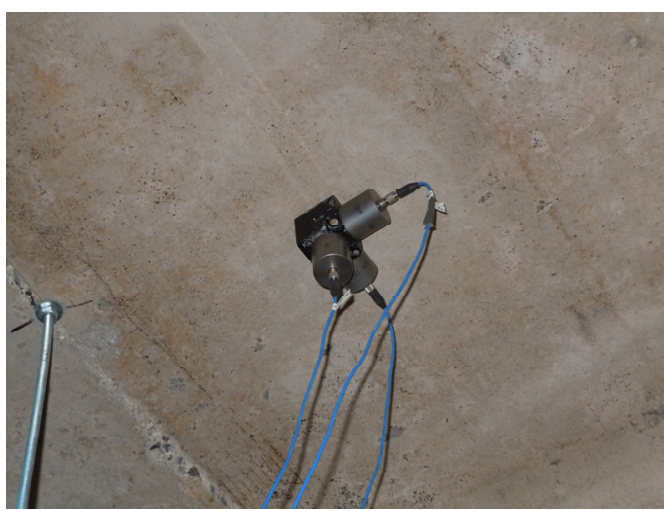

Figure 1. Tri-axial accelerometer cluster installed on floor slab.

Simultaneous vibration testing in adjacent structural bays indicated that the vertical vibrations dissipated with horizontal distance from the operating room location. Approximate calculations indicated that $12 \mathrm{~Hz}$ likely corresponded to the axial natural frequency of the reinforced concrete columns supporting the operating suites.

Based upon these initial results, focus shifted to potential vibration sources within the same structural bay on the floors above and below the operating suite. The continuous nature of the vibrations indicated that the source was most likely mechanical in nature. Due to the complexity of the building systems, a subcontractor was 
retained by the hospital to work with the vibration testing team.

The building automation system was utilized to remotely control the building's mechanical systems. Starting with the floors closest to the fifth floor and radiating outward, the building automation system was utilized to manually control each major mechanical system. Each system was turned off for several minutes and then run at various speeds as applicable. Testing with observation of the new elevators was also performed. The vibration data was observed in semi-real time to determine the impact of each system.

Several nearby mechanical systems were found to have a marginal influence on the operating suite structural vibrations. However, their contribution was not judged to be significant enough to have initiated the complaints. Through the process of elimination, an air handling unit (AHU) on Level 13 of the building was identified to have a significant influence on the vibrations measured on Level 5.

Inspection of the AHU on Level 13 by the mechanical subcontractor indicated that it was not functioning properly. The cooling demand of the building was temporarily redistributed, and the AHU was idled to allow for use of the operating suites. The AHU was repaired and follow-up measurements were collected. The vibrations within the operating suite were reduced by more than $50 \%$ at $12 \mathrm{~Hz}$ after the AHU repairs. Surgeons confirmed that image quality for the floor mounted microscopes was restored.

Analysis of the AHU on Level 13 which had been installed during the vertical expansion indicated that it was not receiving sufficient supply air to meet its programmed output pressure. This caused a galloping phenomenon in which the AHU rocked violently on its supports. The impulses created by this large unit were input into the adjacent column line and carried down to Level 5.

\subsection{Summary and Discussion}

The use of high sensitivity accelerometers in conjunction with a sophisticated building automation system allowed for systematic assessment of the impact of many mechanical systems in a short period of time. The excitation source was eventually identified to be eight levels removed from the surgical suites. Those intermediate floors were primarily used for patient rooms and staff offices. Since the magnitude of the vibrations was below levels perceptible to humans they did not elicit complaints on the higher floors.

While the energy from the AHU was input into the structural system at a much lower frequency, the effect of the discrete impulses from the AHU was to excite the building columns axially which resonated at their natural frequency.

\section{Case Study: Commercial office building human perception vibration source detection}

\subsection{Background}

The management of a high-rise commercial office building in Omaha, Nebraska, USA began receiving complaints of perceptible vibration events in an isolated area of Level 12. The building had been in service for more than 10 years without complaint. Three staff members were complaining in adjacent workspaces along the same column line.

The complaints described intermittent vibration events of varying duration from 5 minutes to 1 hour. The events were described as intense enough to cause mild nausea and to violently rattle computer monitors. The building occupants were asked to record all perceived vibration events over the course of a week and no discernible pattern in timing or duration was evident.

The building management spent approximately two months researching the issue including stationing building maintenance staff in the location of the complaints for entire workdays to make observations and hiring mechanical contractors to inspect and service the building mechanical systems. These measures did not lead to resolution of the issue culminating in our retention.

The 19 story office building is constructed of composite structural steel framing. Each floor has a raised floor system to accommodate electrical and telecommunications wiring and to serve as a chase for the conditioned air supply. The typical floor build-out consisted of one row of closed offices along the perimeter walls with cubicle style workstations throughout the field of the floor.

\subsection{Testing and Results}

Accelerometers were installed on both the structural floor system and the raised floor system at the location of the occupant complaints as shown in Figure 2. The intermittent nature of the vibrations significantly complicated source detection as the timing and duration of detectible events could not be forecast. During periods without vibration events, systematic testing of adjacent mechanical systems including AHUs, elevators, and pumps was conducted. 


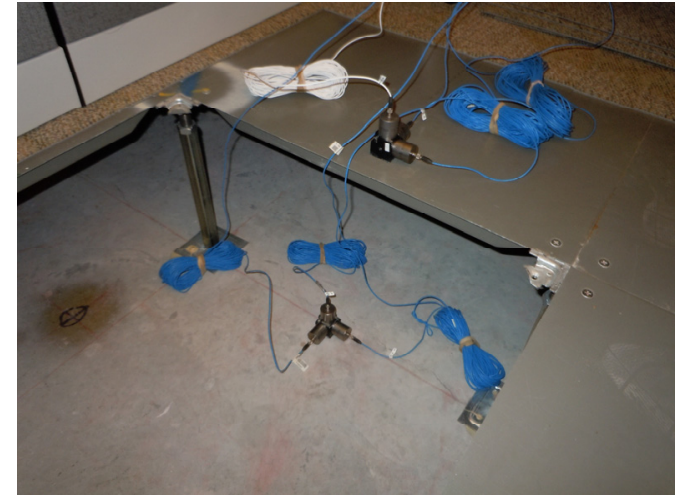

Figure 2. Tri-axial accelerometer clusters installed on the structural floor slab and raised floor system.

While the long duration of some of the vibration events did not correlate well with human excitation of the structure through rhythmic walking, testing was done with various groups of volunteers to produce rhythmic walking excitation along the main corridors. This testing did produce some vibration events close to the limits for human perception of vibrations from AISC DG11; however, the durations produced were inconsistent with the complaints. This testing combined with approximate calculations indicated a natural frequency of about $4 \mathrm{~Hz}$ for the floor system. Coupled with low damping from an open office concept the floor was easily excitable through coordinated movement.

Near the end of the first day of testing two perceptible events were captured lasting approximately 10 minutes each. The results indicated exceedance of the AISC DG11 limits for human perception in an office setting at $4-5 \mathrm{~Hz}$ as shown in Figure 3. Similar monitoring was conducted on Level 11 below. The baseline measurements (excluding bothersome events) were found to be comparable between the two floors.

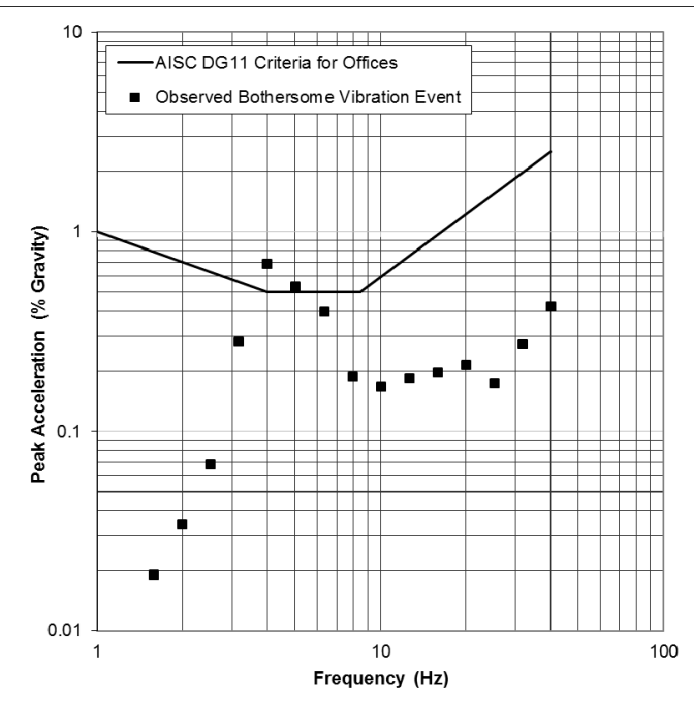

Figure 3. Comparison of bothersome vibration event and AISC DG11 limits for human perception utilizing third octaves analysis.
Having ruled out likely mechanical systems and group walking, focus shifted to observing localized staff behaviour. Eventually it was observed that a staff member several workstations removed from the area of complaints was vigorously bouncing/wobbling both legs while working at his computer. Further observation indicated that times of extreme activity by this staff member corresponded to perceptible events. He was observed to be bouncing/wobbling each leg at approximately $2 \mathrm{~Hz}$ in a staggered fashion very closely matching the $4 \mathrm{~Hz}$ natural frequency of the floor system.

After hours when the staff had left for the day the observed behaviour was recreated and compared with the results obtained earlier in the day as shown in Figure 4.

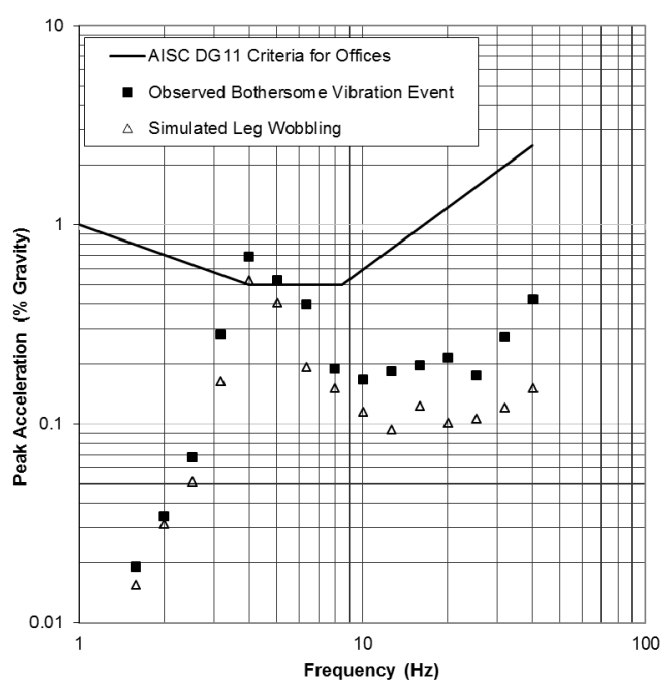

Figure 4. Comparison of bothersome vibration event, simulated leg bouncing activities and AISC DG11 limits for human perception utilizing third octaves analysis.

\subsection{Summary and Discussion}

After the results of the study were shared with building management, the human resources department was able to establish that the start date of the staff member found to be exciting the floor corresponded to the exact date of the first vibration complaint. The staff member was alerted to the impact of his leg bouncing/wobbling and the vibration events ceased. The workstations directly adjacent to the staff member exciting the floor were vacant and the complaining staff members and the leg bouncer worked for different internal departments and therefore never interacted.

In this case the use of instrumentation and deductive reasoning was able to rule out the mechanical systems and the likelihood of transmission from other floors. By focusing on the areas immediately adjacent to the complaints the source was discovered. The varied meeting schedule of the leg bouncing staff member and other human factors accounted for the random distribution of events and durations. 
Like most composite steel structures, the floors within this building were highly susceptible to rhythmic vibrations due to a low natural frequency and low damping. As observed during the testing rhythmic walking could occasionally reach perceptible limits but did not incite complaints due primarily to the intermittency and short durations of walking induced events. The raised floor system was found to amplify the vibration levels slightly at the location of the staff complaints.

\section{Case Study: Hospital human perception vibration source detection}

\subsection{Background}

Several tenants within a low-rise hospital building began to complain of intermittent perceptible vibration events within their workspaces. The complaints emanated from one suite on the fifth and highest level of the hospital. The complaints continued for more than 6 months without resolution. During this time the building management worked to troubleshoot the complaints and tested and serviced many of the building mechanical systems.

Over the course of many months the complaining staff maintained vibration event logs. Review of this data indicated that while the events were seemingly random a greater density of events occurred in the early morning and in the middle of the afternoon. The events were reported to be frequent and closely spaced at times but fleeting in duration, lasting only a couple of seconds. During active periods as many as 25 events would be reported within an hour.

The building management retained a geotechnical testing company to perform vibration measurements. These measurements were collected using a geophone based system capable of measuring and recording discrete impact events. The monitoring was conducted passively over several days with the results collected after conclusion of the monitoring period. While this testing confirmed the presence of intermittent perceptible events, the lack of time history plots and visual observations during the monitoring period created a lack of context for use of the data to determine the likely source(s).

The effected wing of the hospital consisted of 5 levels of underground parking and five occupied levels above grade. The subterranean parking levels are composed of reinforced concrete flat slab construction. The occupied levels are constructed of composite structural steel floor slabs.

\subsection{Testing and Results}

Accelerometers were installed within the 3 offices where the complaining staff members worked. The pattern of reported vibration events initially indicated the possibility of human walking or rhythmic excitation of the slab or cycling of mechanical equipment that runs intermittently. The location of the complaints was a private clinic on a floor with low foot traffic and many closely spaced full height partitions. Synchronized walking activities failed to generate significant vibrations in large part due to the lack of continuous hallways in which excitation could occur.

As indicated by the previous event logs many events were measured during the initial testing. These events correlated with creaking of the suspended ceiling system which heightened the level of concern felt by the occupants. The vibrations were found to have a peak result at $10 \mathrm{~Hz}$.

A significant number of mechanical systems were cycled with the help of the building staff and none were found to correlate with the vibration events on the fifth floor. Testing was next moved to the fourth floor where no complaints had been registered for comparative purposes. The results of this testing indicated similar vibration events as those observed on the fifth floor. While conducting the testing staff members on the fourth floor acknowledged feeling vibration events occasionally. None of the staff on this level had complained to building management. It is likely that a key factor involved the use of these spaces on the fourth floor. The affected areas were infrequently used exam rooms on the fourth floor in comparison to continually occupied offices on the fifth floor.

Once it was determined that the vibrations were not unique to the fifth floor, observations and testing spread out vertically centered around the columns adjacent to the location of the complaints on the fifth floor. During this process a speed bump was observed adjacent to the column line on the top level of the subterranean parking garage as shown in Figure 5. Observation of the garage traffic and simultaneous review of the vibration data on the fifth floor confirmed the link. The vibration events were correlated with vehicles traversing the speed bumps at a high rate of speed. Vehicles traveling as slow speeds did not produce noticeable events.

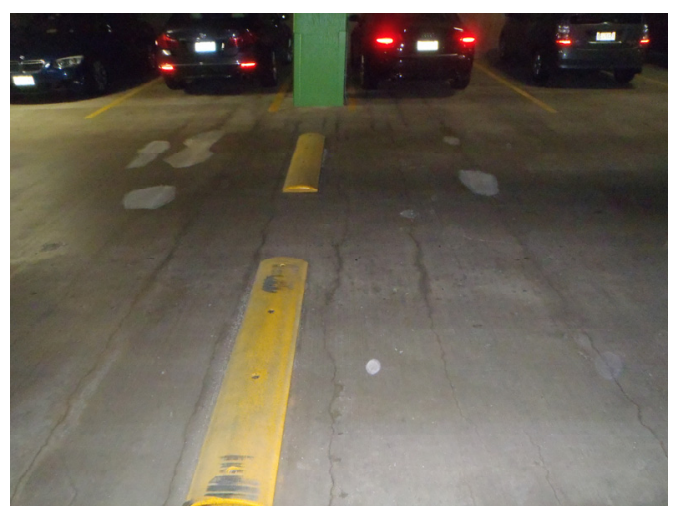

Figure 5. Speed bumps adjacent to the column line of interest.

After presenting the data to the building management, the modular speed bumps were removed from the garage slab and the testing was continued for another working day. The results of the monitoring without the speed bump confirmed that the perceptible vibration events had been eliminated as shown in Figure 6. 


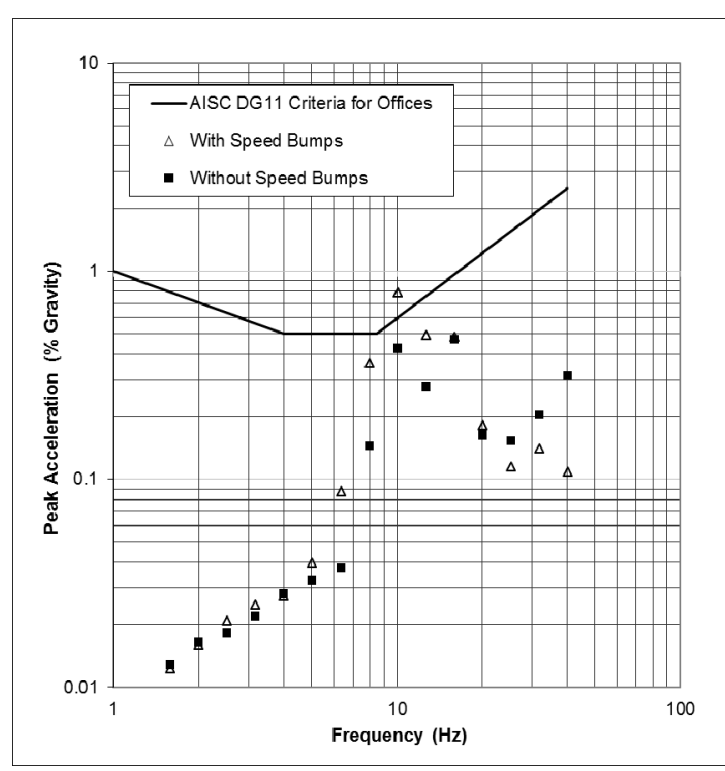

Figure 6. Comparison of vibration levels with and without the speed bumps installed with AISC DG11 limits for human perception utilizing third octaves analysis.

\subsection{Summary and Discussion}

Initial reports that the vibration events were confined to the fifth floor turned out to be misleading. Further review of the building layout indicated that the different uses of that structural bay on each floor contributed to the lack of detection on the lower levels. On the fifth floor staff were assigned to quiet offices where they spent the majority of the working day; therefore they were easily annoyed by frequent and closely spaced vibration events. On the fourth floor that structural bay was occupied by lightly used exam rooms which were shared by many staff members who used them intermittently. On the second and third levels the structural bay was part of the intensive care unit where patients are often unconscious or severely compromised. On the first floor the structural bay was part of an entrance lobby where people passed through but did not linger.

Research of the issue confirmed that the speed bumps had been installed around the time of the first complaints. Ironically, the speed bumps had been installed in an attempt to quell vehicular speed in the garage. The lower levels of the garage were utilized for staff parking and many were observed driving very fast through the garage early in the morning and in the afternoon after their shift. During the middle of the day visiting patients comprised most of the traffic and generally travelled at slower speeds.

Systematic evaluation of the problem and trial and error allowed for triangulation of the vibration source. While documented complaints always serve as an important first clue, it is often necessary to test other areas of the structure to provide appropriate context to the problem.

\section{Conclusions}

These three case studies present the methodology used to determine the source of bothersome vibrations in complex occupied structures. The use of active semireal time vibration monitoring with a trial and error approach to eliminate potential sources allows for source determination. While each problem is unique some tips for tackling source determination problems are included herein:

1. Interview building staff to understand the nature of the complaints. While useful consider the limitations of staff accounts and the variability of human perception.

2. Record a bothersome event(s) to understand the characteristics of the vibrations including magnitude, frequency, and duration.

3. Assess the likelihood of different excitation sources based on the event pattern (continuous, intermittent, or periodic). This can help prioritize systems to assess.

4. Seek to understand the vibration profile in surrounding areas including adjacent structural bays and adjacent floors. This can help establish the direction in which the events are radiating.

5. Systematically begin to test and eliminate potential sources starting at the location of concern and radiating outward (either horizontally or vertically).

6. Perform testing after pacifying the identified source(s) to provide documentation of the impact of that source.

\section{References}

1. T.M. Murray, D.E. Allen, E.E. Ungar, American Institute of Steel Construction; Design Guide 11: Floor Vibrations Due to Human Activity, (2003) 